\title{
VEGF is indirectly associated with NO production and acutely increases in response to hyperglycaemia ${ }^{1}$
}

\author{
Mario Siervo ${ }^{*}$, Virginia Tomatis ${ }^{\dagger}$, Blossom C. M. Stephan $^{\ddagger}$, Martin Feelisch $^{\S}$ and Les J. C. Bluck ${ }^{\dagger}$ \\ *Human Nutrition Research Centre, Institute for Ageing and Health, Newcastle University, Newcastle On Tyne, UK, \\ ${ }^{\dagger}$ MRC Human Nutrition Research, Elsie Widdowson Laboratory, Cambridge, UK, ${ }^{\ddagger}$ Department of Public Health and Primary \\ Care, University of Cambridge, Cambridge, UK, ${ }^{5}$ Warwick Medical School, University of Warwick, Coventry, UK
}

\begin{abstract}
Background Increased levels of vascular endothelial growth factor (VEGF) have been observed in patients with metabolic syndrome (MetS). Nitric oxide (NO) formation is reduced in MetS, but its relationship to VEGF production remains poorly defined. We evaluated the association between VEGF/NO synthesis and insulin sensitivity in obese subjects and investigated the secretory response of VEGF to an acute elevation of glucose.
\end{abstract}

Materials and methods Seven healthy normal-weight subjects, seven obese subjects without MetS and seven obese subjects with MetS were recruited. Anthropometry, body composition and cardiometabolic functions (blood pressure, glucose, insulin, triglycerides, total cholesterol, HDL-C and VEGF) were measured, and a novel stable isotope method was used to assess in vivo rates of NO production. A frequent sampling intravenous glucose tolerance test was performed to study the dynamics of VEGF release.

Results Fasting VEGF levels were significantly higher in the two obese groups compared to the control group $(P$ for trend $=0.02)$, but the difference was not significant after adjustment for age. Vascular endothelial growth factor levels were associated with systolic blood pressure $(\rho=0.54 ; P=0.01)$ and NO production $(\rho=-0.44$; $P=0.04)$. Vascular endothelial growth factor levels increased in response to acute hyperglycaemia in normalweight and obese subjects $(P<0.001)$.

Conclusions Vascular endothelial growth factor levels rapidly increase during hyperglycaemia and are inversely related to NO production at steady state. The potential link between the acute secretion of VEGF and atherosclerotic risk in subjects with poorly controlled glycaemia as well as the potential of lowering elevated VEGF levels by increasing NO production and/or availability warrants further investigation.

Keywords Hyperglycaemia, nitric oxide, obesity, vascular endothelial growth factor.

Eur J Clin Invest 2012; 42 (9): 967-973

\section{Introduction}

Angiogenesis is involved in the formation of new vascular networks in physiology (embryo/organogenesis, pregnancy, wound healing) and pathology (cancer, psoriasis) [1], and de-novo formation and remodelling of blood vessels are important events in the atherosclerotic process. Current research is investigating the role of angiogenesis as a risk factor for atherosclerosis and excess adiposity [2,3].

Vascular endothelial growth factor levels were directly associated with body mass index (BMI) but not with insulin

\footnotetext{
${ }^{1}$ The material presented in this manuscript is original and has not been submitted for publication elsewhere while under consideration for European Journal of Clinical Investigation.
}

sensitivity in obese and normal-weight subjects [4]. Plasma VEGF levels were significantly associated with features of the metabolic syndrome (MetS) such as BMI, waist circumference, blood pressure and inflammation [5,6].

In vivo rates of nitric oxide (NO) synthesis are lower in metabolic disorders associated with insulin resistance [7]. In addition, we have recently demonstrated a reduction in NO production in obese subjects with MetS [8] and a direct association with insulin sensitivity [9], suggesting that VEGF may be inversely associated with $\mathrm{NO}$ production and insulin sensitivity. Here, we investigated this hypothetical association by comparing levels of VEGF in obese subjects with and without MetS to normal-weight controls. In addition, the acute secretory 
response of VEGF to an intravenous glucose bolus was investigated in controls and obese subjects.

\section{Methods}

\section{Subjects}

Fourteen obese subjects (BMI: $30-40 \mathrm{~kg} / \mathrm{m}^{2}$ ) and seven normalweight subjects (BMI: $18 \cdot 5-25 \cdot 0 \mathrm{~kg} / \mathrm{m}^{2}$ ) were recruited. Seven obese subjects had three or more criteria for the metabolic syndrome. All subjects gave written informed consent before participating in the study. The study was approved by the Cambridgeshire four Research Ethics Committee. Participants were excluded if they had any condition or were taking any medication that may have interfered with the analyses. A full description of the exclusion criteria is reported in the Online Supporting Information. Reporting of the study conforms to STROBE statement along with references to STROBE and the broader EQUATOR guidelines [10].

\section{Screening visit}

Body mass index was calculated, with weight and height measured to the nearest $0 \cdot 1 \mathrm{~kg} / \mathrm{cm}$. Waist circumference was measured in triplicate. Body fat was measured using a leg-to-leg bio-impedance analyser (Tanita Corporation, Japan). Resting blood pressure was measured in triplicate using an automated sphygmomanometer (Dynamap, Critikon, UK), with a larger cuff for obese subjects. Blood and kidney function tests were performed after an overnight fast to ensure that participants met the inclusion criteria. The diagnosis of MetS was made according to the recent criteria proposed in a joint statement by the major diabetes, obesity and heart organisations [11], which requires at least three of the following criteria be fulfilled: waist circumference $(\mathrm{WC}) \geq 102 \mathrm{~cm}$ (man), $\geq 88 \mathrm{~cm}$ (woman); triglycerides (TG) $\geq 1.7 \mathrm{mM}$; high-density lipoproteins (HDL-C) $<1.3 \mathrm{mM}$ (man), < 1.1 mM (woman); high blood pressure $\geq 130 / 85 \mathrm{mmHg}$; fasting plasma glucose $\geq 5.6 \mathrm{mM}$.

\section{Frequent sampling intravenous glucose tolerance test (FSIVGTT)}

Subjects arrived in the morning to the research unit after they had fasted for at least $12 \mathrm{~h}$ and followed the low $\mathrm{NO}_{3}^{-}$diet for $24 \mathrm{~h}$ as part of the Salivary Oral Nitrate Test (S-ONT) used for the measurement of NO production [8]. Two cannulae were inserted in the antecubital vein, one in each arm. Three blood samples were taken before the administration of glucose to measure baseline insulin and glucose levels. An intravenous glucose dose (1.75 g of [6,6]- ${ }^{2} \mathrm{H}_{2}$-glucose to $19 \mathrm{~g}$ unlabelled glucose in the form of a $50 \%$ aqueous solution) was then given over $1 \mathrm{~min}$. Twenty minutes after glucose administration, $201 \mathrm{mU}$ of Human Actrapid insulin was given. A total of 30 blood samples were taken at $-10,-5,-1,1,2,3,4,5,6,8,10,13,15,20,25,30$,
$35,40,50,60,70,80,90,100,120,140,160,180,210$ and $240 \mathrm{~min}$ relative to the administration of glucose. Insulin sensitivity $(\mathrm{Si})$ was characterised by the minimal model as defined by Bergman et al. [12]. The plasma glucose and insulin concentrations during the FSIVGTT are showed in Figure S1 of the online Data S1. After the FSIVGTT, subjects were served a very low $\mathrm{NO}_{3}^{-}$meal as part of the oral nitrate test protocol (ONT). A description of the meals and the low $\mathrm{NO}_{3}^{-}$diet provided have been previously described [8] and it is reported in the Online Supporting Information.

\section{Salivary oral nitrate test (S-ONT)}

After the low $\mathrm{NO}_{3}^{-}$meal, each participant was instructed not to eat and to drink only low $\mathrm{NO}_{3}^{-}$bottled water (Buxton) until the following morning (18-h fasting period). Four hours after the meal, a pre-dose saliva sample was collected and a dose of $4 \mathrm{mg}$ of $\mathrm{Na}^{15} \mathrm{NO}_{3}^{-}$was given as a drink in $100 \mathrm{~mL}$ of distilled water. Each subject was asked to provide another saliva sample $2 \mathrm{~h}$ later. All subsequent saliva samples were collected by the volunteer at home using pre-labelled containers. The protocol for the collection of saliva samples has been previously described [8]. The final saliva sample was collected $18 \mathrm{~h}$ after the meal on the following morning. NO production was estimated by analysing the isotopic decay in saliva of an oral dose of labelled $\mathrm{NO}_{3}^{-}$using an exponential function for a single compartment. Data were described using a semilogarithmic plot, and the slope and intercept of the regression line were used to derive NO synthesis, as previously described [8].

\section{Materials}

Trifluoroacetic anhydride (TFAA), sodium hydroxide and mesityline were obtained from Sigma-Aldrich Company Ltd, Poole, Dorset, UK. All the stable isotopically labelled compounds, ${ }^{15} \mathrm{~N}$-sodium nitrate $(98 \%+),[6,6]-{ }^{2} \mathrm{H}_{2}$-glucose $(98 \%+)$ were obtained from Cambridge Isotope Laboratories Inc, Andover, MA, USA.

\section{Nitrate measurements}

Salivary nitrate concentrations were measured using a commercial 96-well kit (Cayman Chemical Co, Ann Arbor, MI, USA). ${ }^{15} \mathrm{~N}$ enrichment of nitrate was measured by gas chromatography mass spectrometry (GC/MS) following derivatisation of saliva samples with mesitylene and TFAA as catalyst to give a single product, nitromesitylene (1,3,5-trimethyl nitrobenzene), as previously described [13].

\section{Glucose enrichment measurement}

Plasma glucose enrichments were analysed by GC/MS after derivatisation with fluorinated methyl boronic acid, as previously described [14]. 


\section{Clinical biochemistry}

Blood (full blood count, glucose, insulin, Hb1Ac), lipid profile (HDL-C, LDL-C, triglycerides and total cholesterol), $C$ reactive protein (CRP) and kidney function tests (urea, creatinine) were measured using validated methods. Plasma asymmetric dimethylarginine (ADMA) levels were measured by competitive ELISA (Immunodiagnostik, Belsham, Germany). Plasma VEGF concentrations were measured using an enzyme-linked immunosorbent assay (Quantikine ${ }^{\mathrm{TM}}$; R\&D Systems, Minneapolis, MN, USA).

\section{Statistical analysis}

Some of the results presented in this manuscript have been reported elsewhere [8]. Data are shown as mean and standard deviation (SD). The Kruskal-Wallis and the Mann-Whitney test were used to test for differences between groups. The Friedman test was used to test within-group differences in VEGF levels during acute hyperglycaemia. Analysis of covariance for repeated measures was used to assess VEGF changes during acute hyperglycaemia after including age in the model as covariate. Time ( $\mathrm{T}$ ) was the repeated measure factor, and group $(G)$ was entered as the between-subject factor. The interaction term $(T \times G)$ was built to assess between-group differences in VEGF responses during hyperglycaemia. Variables entered into the model were transformed using the rank transformation method to account for the small sample size and deviation from normality distribution [15]. The Spearman rank correlation (unadjusted and age-adjusted) was used to assess the association between VEGF and cardiovascular and metabolic parameters. All statistical analyses were carried out using SPSS 16 for Windows (SPSS Inc., Chicago, IL, USA). The significance level was set at 0.05.

\section{Results}

The baseline characteristics of the study participants are shown in Table 1. Subjects in the control group were significantly younger and had a better overall metabolic profile than those in either obese group. There were no differences between groups for kidney function, glucose, diastolic blood pressure and HDL-C. Plasma levels of insulin, triglycerides and total cholesterol were significantly higher in the obese group with MetS. The control group had higher values of Si compared to the two obese groups. In contrast, rates of NO synthesis were significantly lower in the obese group with MetS compared to the control and obese without MetS groups. Differences in NO production remained significant after age adjustment $(P=0 \cdot 006)$. Fasting VEGF levels were significantly higher in the two obese groups compared to the control group ( $P$ for trend $=0.02$ ), but the differ- ence was no longer significant after adjustment for age (Table 1).

Vascular endothelial growth factor concentrations were directly correlated with age, BMI, WC, fat mass (FM), SBP and fasting insulin and inversely related to insulin sensitivity and NO production. After age adjustment, only SBP and NO synthesis remained significantly associated with VEGF levels. Vascular endothelial growth factor levels were not significantly associated with biomarkers of endothelial dysfunction (ADMA) and inflammation (CRP) (Table 2).

Plasma VEGF levels rapidly increased in response to rising plasma glucose levels during the initial phase of the FSIVGTT. The increase in VEGF levels remained significant after age adjustment $(P<0.001)$, and the proportional changes relative to baseline after $19 \mathrm{~min}$ were $67 \%$ for the control group $(P=0.003), 70 \%$ for the obese without MetS $(P=0.003)$ and $39 \%$ for the obese group with MetS $(P=0.54)$. The interaction term $(\mathrm{T} \times \mathrm{G})$ in the adjusted model was not significant $(P=0.09)$, which indicates a similarity in VEGF responses to hyperglycaemia between the three groups (Fig. 1).

\section{Discussion}

Obese subjects with MetS had significantly lower levels of NO production and insulin sensitivity compared to obese subjects without MetS and normal-weight subjects. Fasting NO and SBP were significantly correlated with VEGF levels, which may suggest interplay of these factors in the development of endothelial dysfunction in obesity. Hyperglycaemia induced a rapid increase in VEGF levels, and the responses were greater in normal-weight and obese subjects without MetS. The difference between the two obese groups may be related to the higher degree of endothelial dysfunction associated with MetS, potentially translating into slower signal transduction and a delayed VEGF secretory response. This assumption could not be experimentally tested in our protocol as an insulin bolus was administered at $20 \mathrm{~min}$, confounding the effects of hyperglycaemia on VEGF secretion (no VEGF levels were measured past $20 \mathrm{~min}$ ).

This study revealed a reciprocal association between $\mathrm{NO}$ production and VEGF levels using for the first time a validated stable isotope method for the measurement of in vivo rates of whole-body NO production [8]. A reciprocal relationship between NO and VEGF production has been observed in cellular models [16] but these results are not consistent with human studies $[17,18]$. Specifically, two studies have reported lower plasma nitrate levels, used as an index of NO production and decreased VEGF levels in obese subjects with MetS [17] and hypertension [18]. The decrease in VEGF levels observed in these two studies contrasts with the increase in VEGF levels observed in subjects with obesity [19], MetS [5], hypertension 
Table 1 Baseline characteristics of the control group and of the two obese groups divided according to the diagnosis of metabolic syndrome (MetS)

\begin{tabular}{|c|c|c|c|c|}
\hline & Controls & Obese without MetS & Obese with MetS & $P$ value \\
\hline$N$ & 7 & 7 & 7 & \\
\hline $\mathrm{M} / \mathrm{F}$ & $3 / 4$ & $1 / 6$ & $3 / 4$ & \\
\hline Age (years) & $27 \cdot 4(10 \cdot 9)$ & $48.0(8.0)$ & $48.0(10 \cdot 7)$ & $0.01^{a, b}$ \\
\hline Weight (kg) & $65 \cdot 2(16 \cdot 2)$ & $95 \cdot 7(11 \cdot 8)$ & $103.9(10 \cdot 7)$ & $0.001^{\mathrm{a}, \mathrm{b}}$ \\
\hline Height (cm) & $171.0(14.0)$ & $166 \cdot 4(9 \cdot 8)$ & $176 \cdot 1(5 \cdot 4)$ & 0.24 \\
\hline Body mass index $\left(\mathrm{kg} / \mathrm{m}^{2}\right)$ & $21.9(2 \cdot 2)$ & $34.5(2 \cdot 3)$ & $33.4(2.9)$ & $0.001^{a, b}$ \\
\hline Waist circumference $(\mathrm{cm})$ & $78.0(10 \cdot 7)$ & $109.9(8.5)$ & $111 \cdot 3(5 \cdot 1)$ & $0.001^{a, b}$ \\
\hline Systolic blood pressure $(\mathrm{mmHg})$ & $106 \cdot 7(8 \cdot 3)$ & $120 \cdot 3(13 \cdot 1)$ & $130 \cdot 1(11 \cdot 6)$ & $0.01^{\mathrm{b}}$ \\
\hline Diastolic blood pressure $(\mathrm{mmHg})$ & $64 \cdot 4(10 \cdot 2)$ & $74 \cdot 2(10 \cdot 2)$ & $76.0(7 \cdot 7)$ & $0 \cdot 16$ \\
\hline Glucose (mmol/L) & $4 \cdot 3(0 \cdot 4)$ & $4.7(0.4)$ & $4.9(0.5)$ & $0 \cdot 20$ \\
\hline Insulin (pmol/L) & $28.8(6 \cdot 8)$ & $48 \cdot 8(18 \cdot 1)$ & $56 \cdot 5(12.5)$ & $0.004^{a, b}$ \\
\hline Triglycerides (mmol/L) & $0.8(0.3)$ & $1 \cdot 1(0 \cdot 3)$ & $1.8(0.4)$ & $0.006^{b}$ \\
\hline HDL-C (mmol/L) & $1.5(0.3)$ & $1.5(0 \cdot 1)$ & $1 \cdot 2(0 \cdot 1)$ & 0.07 \\
\hline Total cholesterol (mmol/L) & $4.4(0.7)$ & $5.9(1 \cdot 2)$ & $6 \cdot 1(1 \cdot 4)$ & $0.05^{\mathbf{b}}$ \\
\hline Creatinine $(\mathrm{mmol} / \mathrm{L})$ & $74.7(11.9)$ & $63.0(6 \cdot 8)$ & $78 \cdot 7(17 \cdot 4)$ & 0.09 \\
\hline Urea (mmol/L) & $4.0(1.3)$ & $4.5(0.6)$ & $5 \cdot 2(0 \cdot 1)$ & $0 \cdot 16$ \\
\hline $\mathrm{CRP}(\mathrm{mg} / \mathrm{L})$ & $0.98(0.63)$ & $3.37(1.66)$ & $2.97(1.90)$ & $0.01^{a, b}$ \\
\hline ADMA $(\mu \mathrm{mol} / \mathrm{L})$ & $0.44(0.07)$ & $0.48(0.06)$ & $0.59(0.08)$ & $0.02^{b, c}$ \\
\hline Insulin sensitivity (pmol/h/L) & $0.012(0.008)$ & $0.0057(0.0026)$ & $0.0052(0.0031)$ & $0.05^{a, b}$ \\
\hline Nitric oxide synthesis $(\mu \mathrm{mol} / \mathrm{kg} / \mathrm{h})$ & $0.63(0.29)$ & $0.49(0.22)$ & $0.21(0.13)$ & $0.009^{b, c}$ \\
\hline $\operatorname{VEGF}(\mathrm{pg} / \mathrm{mL})$ & $11.01(4.09)$ & $20 \cdot 70(9 \cdot 35)$ & $18 \cdot 86(4 \cdot 70)$ & $0.02^{a, b}$ \\
\hline
\end{tabular}

Mean (SD) is shown. Significant results are shown in bold. Kruskal-Wallis test was used to test for differences across the three groups (trends). The Mann-Whitney test was utilised in a post hoc analysis to compare the individual groups. Letters indicate statistical significance between groups $(P<0.05)$. VEGF, vascular endothelial growth factor; CRP, $C$ reactive protein; ADMA, asymmetric dimethylarginine.

${ }^{a}$ Control vs. Obese without MetS.

${ }^{b}$ Control vs. Obese with MetS.

${ }^{\mathrm{c}}$ Obese with MetS vs. Obese without MetS.

[20], type 2 diabetes with and without microvascular complications (nephropathy, retinopathy)[21], coronary heart disease [22] and peripheral artery disease [22]. In diabetic and nondiabetic obese subjects [23] and in hypertensive patient with and without retinopathy [20], flow-mediated dilation of the brachial artery in response to reactive hyperaemia was inversely associated with the levels of VEGF. Valabhji et al. [24] showed that elevated VEGF levels in patients with type 1 diabetes correlated with decreased carotid artery distensibility. Moreover, VEGF directly correlated with carotid-to-intima media thickness in 909 healthy subjects $\left(\mathrm{BMI}=26.5 \pm 4 \cdot 2 \mathrm{~kg} / \mathrm{m}^{2}\right)$ from the Salzburg Atherosclerosis Prevention Program in Subjects at High Individual Risk (SAPHIR)[25]. Thus, our results are in agree- ment with a robust direct association between VEGF and endothelial dysfunction, suggesting potential new therapeutic avenues targeting elevated VEGF levels by enhancing NO production and/or availability.

The VEGF response to controlled hyperglycaemia in the obese without MetS and normal-weight controls suggests that endothelial cells are sensitive to acute changes in metabolic fluxes, which may be a physiological response to cellular changes in redox status and related to increased nutrient uptake. Insulin stimulates VEGF expression [26], and a recent study has demonstrated a role of VEGF-B in endothelial fatty acids uptake [27]. A significant direct correlation between plasma VEGF concentration and $\mathrm{HbA} 1 \mathrm{c}$ has been observed in 
Table 2 Unadjusted and age-adjusted correlation between vascular endothelial growth factor (VEGF, pg/mL) and the main measures of metabolic and cardiovascular function

\begin{tabular}{|c|c|c|c|c|}
\hline & \multicolumn{2}{|l|}{ Unadjusted } & \multicolumn{2}{|l|}{ Adjusted for age } \\
\hline & Rho coefficient & $P$ value & Rho coefficient & $P$ value \\
\hline Age (years) & 0.56 & 0.008 & - & - \\
\hline Body mass index $\left(\mathrm{kg} / \mathrm{m}^{2}\right)$ & 0.56 & 0.008 & $0 \cdot 40$ & 0.07 \\
\hline Waste circumference $(\mathrm{cm})$ & 0.63 & 0.002 & $0 \cdot 42$ & 0.06 \\
\hline Systolic blood pressure (mmHg) & 0.72 & $<0.001$ & 0.54 & 0.01 \\
\hline Diastolic blood pressure $(\mathrm{mmHg})$ & $0 \cdot 24$ & $0 \cdot 28$ & $-0 \cdot 15$ & 0.54 \\
\hline Glucose (mmol/L) & 0.32 & $0 \cdot 14$ & 0.02 & 0.93 \\
\hline Insulin (pmol/L) & 0.54 & 0.01 & $0 \cdot 36$ & $0 \cdot 11$ \\
\hline Cholesterol (mmol/L) & 0.04 & 0.83 & $-0 \cdot 30$ & $0 \cdot 18$ \\
\hline Triglycerides (mmol/L) & $0 \cdot 22$ & $0 \cdot 31$ & -0.05 & 0.83 \\
\hline $\mathrm{HDL}-\mathrm{C}(\mathrm{mmol} / \mathrm{L})$ & -0.22 & $0 \cdot 31$ & $-0 \cdot 14$ & 0.55 \\
\hline $\mathrm{CRP}(\mathrm{mg} / \mathrm{L})$ & $0 \cdot 32$ & $0 \cdot 15$ & $0 \cdot 12$ & $0 \cdot 62$ \\
\hline ADMA $(\mu \mathrm{mol} / \mathrm{L})$ & $0 \cdot 17$ & $0 \cdot 46$ & $-0 \cdot 14$ & 0.55 \\
\hline NO synthesis $(\mu \mathrm{mol} / \mathrm{h} / \mathrm{kg})$ & -0.50 & 0.02 & -0.44 & 0.04 \\
\hline Insulin sensitivity (pmol/h/L) & -0.49 & 0.02 & -0.28 & 0.22 \\
\hline
\end{tabular}

Spearman rank correlation was used to test the strength of the association between VEGF and the other variables. Significant results are highlighted in bold.

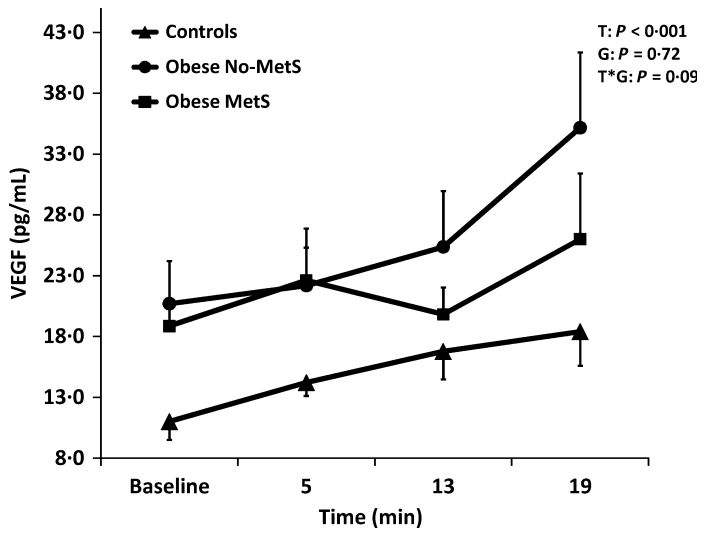

Figure 1 Acute response of vascular endothelial growth factor (VEGF) to controlled hyperglycaemia in controls and obese subjects with and without metabolic syndrome (MetS). Analysis of covariance for repeated measures was used to assess VEGF changes during acute hyperglycaemia after including age in the model as covariate. Time (T) was the repeated measure factor, and group (G) was entered as the between-subject factor. The interaction term $(T \times G)$ was built to assess between-group differences in VEGF responses during hyperglycaemia. Error bars are SE. patients with diabetes [21], suggesting that chronic hyperglycaemia may increase VEGF secretion and that reduction in elevated levels of VEGF may be possible with improved glycaemic control [28]. However, Loebig et al. [4] showed a lack of association of VEGF with insulin sensitivity in normal-weight and obese subjects, and VEGF levels remained unchanged in patients with type 1 diabetes after $210 \mathrm{~min}$ of hyperglycaemia (12.0 $\mathrm{mM})$ and normoglycaemic $(5 \cdot 0 \mathrm{mM})$ clamps with high $(120 \mathrm{mU} / \mathrm{kg} / \mathrm{h})$ and standard insulin infusions $(30 \mathrm{mU} / \mathrm{kg} / \mathrm{h})$, respectively [29].

The cross-sectional design and the small sample size were important limitations of this study, and the results should therefore be interpreted with caution. In addition, a significantly younger control group may have confounded the association. We purposely chose this group as the reference population to remove potential age-related changes in hormonal and cardiometabolic functions to evaluate the deviation of NO production measured in obese, insulin-resistant individuals from optimal, physiological rates. The analyses were adjusted for age to account for the potential confounding effect. Nevertheless, ours is the first report of a reciprocal relationship between NO production, as measured using stable isotope tracers, and plasma VEGF levels and the magnitude of acute increases in VEGF secretion during hyperglycaemia. These 
results warrant further investigation in larger clinical studies, in particular with regard to their relevance for an enhanced atherosclerotic risk in obesity and in patients with diabetes with poorly controlled glycaemia.

\section{Acknowledgements}

We thank Aurelie Tireford, Shelley Parker, Owen Mugridge, Sarah Jackson and Susan Bryant for their help. This study was funded by the Medical Research Council.

\section{Conflicts of interest}

None declared.

\section{Contributions}

MS contributed to the design of the study and collection of the data, analysis of the samples and wrote the manuscript. VT contributed to the sample analysis and to the critical revision of the manuscript. BCM contributed to the statistical analysis and to the critical revision of the manuscript. MF contributed to the interpretation of the results and to the critical revision of the manuscript. LCJB designed the study and revised the manuscript.

\section{Address}

Human Nutrition Research Centre, Institute for Ageing and Health, Newcastle University, Biomedical Research Building, Campus for Ageing and Vitality, Newcastle on Tyne, NE4 5PL, UK (M. Siervo); MRC Human Nutrition Research, Elsie Widdowson Laboratory, Fulbourn Rd, Cambridge, CB1 9NL, UK (V. Tomatis, L. J. C Bluck); Department of Public Health and Primary Care, University of Cambridge, University Forvie Site, Robinson Way, Cambridge, CB2 0SR, UK (B. C. M. Stephan); Warwick Medical School, University of Warwick, Gibbet Hill Road, Coventry, CV4 7AL, UK (M. Feelisch). Correspondence to: Dr Mario Siervo, Human Nutrition Research Centre, Institute for Ageing and Health, Newcastle University, Biomedical Research Building, Campus for Ageing and Vitality, Newcastle on Tyne, NE4 5PL, UK.

Tel.: +44 (0) 0191 2481140; fax: +44(0)191 248 1101; e-mail: mario.siervo@newcastle.ac.uk

Received 3 December 2011; accepted 10 April 2012

\section{References}

1 Birk DM, Barbato J, Mureebe L, Chaer RA. Basic science review: current insights on the biology and clinical aspects of VEGF regulation. Vasc Endovascular Surg 2009;42:517-30.

2 Cao Y. Adipose tissue angiogenesis as a therapeutic target for obesity and metabolic diseases. Nat Rev Drug Discov 2010;9:107-15.

3 Kimura K, Hashiguchi T, Deguchi T, Horinouchi S, Uto T, Oku H et al. Serum VEGF-as a prognostic factor of atherosclerosis. Atherosclerosis 2007:194:182-8.
4 Loebig M, Klement J, Schmoller A, Betz S, Heuck N, Schweiger U et al. Evidence for a relationship between VEGF and BMI independent of insulin sensitivity by glucose clamp procedure in a homogenous group healthy young men. PLoS ONE 2010;5:e12610.

5 Siervo M, Ruggiero D, Sorice R, Nutile T, Aversano M, Stephan BC et al. Angiogenesis and biomarkers of cardiovascular risk in adults with metabolic syndrome. J Intern Med 2010;268:338-47.

6 Siervo M, Ruggiero D, Sorice R, Nutile T, Aversano M, Iafusco M et al. Body mass index is directly associated with biomarkers of angiogenesis and inflammation in children and adolescents. Nutrition 2012;28(3):262-6.

7 Siervo M, Stephan BC, Feelisch M, Bluck LJ. Measurement of in vivo nitric oxide synthesis in humans using stable isotopic methods: a systematic review. Free Radic Biol Med 2011;51:795-804.

8 Siervo M, Jackson SJ, Bluck LJ. In-vivo nitric oxide synthesis is reduced in obese patients with metabolic syndrome: application of a novel stable isotopic method. J Hypertens 2011;29:1515-27.

9 Siervo M, Bluck LJC. In vivo nitric oxide synthesis, insulin sensitivity and asymmetric dimethylarginine in obese subjects without and with metabolic syndrome. Metabolism 2011;doi. org/10.1016/j.metabol.2011.10.003.

10 Simera I, Moher D, Hoey J, Schulz KF, Altman DG. A catalogue of reporting guidelines for health research. Eur J Clin Invest 2010;40: 35-53.

11 Alberti KGMM, Eckel RH, Grundy SM, Zimmet PZ, Cleeman JI, Donato KA et al. Harmonizing the metabolic syndrome: a joint interim statement of the International Diabetes Federation Task Force on Epidemiology and Prevention; National Heart, Lung, and Blood Institute; American Heart Association; World Heart Federation; International Atherosclerosis Society; and International Association for the Study of Obesity. Circulation 2009;120:1640-5.

12 Bergman RN, Ider YZ, Bowden CR, Cobelli C. Quantitative estimation of insulin sensitivity. Am J Physiol Endocrinol Metab 1979;236: E667-77.

13 Jackson S, Siervo M, Persson E, McKenna LM, Bluck LJC. A novel derivative for the assessment of urinary and salivary nitrate using gas chromatography/mass spectrometry. Rapid Commun Mass Spectrom 2008;22:4158-64.

14 Jackson SJ, Waterhouse JS, Bluck LJC. A single glucose derivative suitable for gas chromatography/mass spectrometry and gas chromatography/combustion/isotope ratio mass spectrometry. Rapid Commun Mass Spectrom 2007;21:3123-8.

15 Conover WJ, Iman RL. Analysis of covariance using the rank transformation. Biometrics 1982;38:715-24.

16 Kimura H, Esumi H. Reciprocal regulation between nitric oxide and vascular endothelial growth factor in angiogenesis. Acta Biochim Pol 2003;50:49-59.

17 Barylski M, Kowalczyk E, Banach M, Ciecwierz J, Pawlicki L, Kowalski J. Plasma total antioxidant activity in comparison with plasma NO and VEGF levels in patients with metabolic syndrome. Angiology 2009;60:87-92.

18 Vyzantiadis T, Karagiannis A, Douma S, Harsoulis P, Vyzantiadis A, Zamboulis C. Vascular endothelial growth factor and nitric oxide serum levels in arterial hypertension. Clin Exp Hypertens 2006;28:603-9.

19 Silha JV, Krsek M, Sucharda P, Murphy LJ. Angiogenic factors are elevated in overweight and obese individuals. Int J Obes (Lond) 2005;29:1308-14.

20 Tsai WC, Li YH, Huang YY, Lin CC, Chao TH, Chen JH. Plasma vascular endothelial growth factor as a marker for early vascular damage in hypertension. Clin Sci (Lond) 2005;109:39-43. 
21 Kakizawa $\mathrm{H}$, Itoh $\mathrm{M}$, Itoh $\mathrm{Y}$, Imamura S, Ishiwata $\mathrm{Y}$, Matsumoto T et al. The relationship between glycemic control and plasma vascular endothelial growth factor and endothelin-1 concentration in diabetic patients. Metabolism 2004;53:550-5.

22 Blann AD, Belgore FM, McCollum CN, Silverman S, Lip PL, Lip GY. Vascular endothelial growth factor and its receptor, Flt-1, in the plasma of patients with coronary or peripheral atherosclerosis, or Type II diabetes. Clin Sci (Lond) 2002;102:187-94.

23 Doupis J, Rahangdale S, Gnardellis C, Pena SE, Malhotra A, Veves A. Effects of diabetes and obesity on vascular reactivity, inflammatory cytokines, and growth factors. Obesity (Silver Spring) 2011;19:729-35.

24 Valabhji J, Dhanjil S, Nicolaides AN, Elkeles RS, Sharp P. Correlation between carotid artery distensibility and serum vascular endothelial growth factor concentrations in type 1 diabetic subjects and nondiabetic subjects. Metabolism 2001;50:825-9.

25 Sandhofer A, Tatarczyk T, Kirchmair R, Iglseder B, Paulweber B, Patsch JR et al. Are plasma VEGF and its soluble receptor sFlt-1 atherogenic risk factors? Cross-sectional data from the SAPHIR study Atherosclerosis 2009;206:265-9.

26 Treins C, Giorgetti-Peraldi S, Murdaca J, Semenza GL, Van Obberghen E. Insulin stimulates hypoxia-inducible factor 1 through a phosphatidylinositol 3-kinase/target of rapamycin-dependent signaling pathway. J Biol Chem 2002;277:27975-81.
27 Hagberg CE, Falkevall A, Wang X, Larsson E, Huusko J, Nilsson I et al. Vascular endothelial growth factor B controls endothelial fatty acid uptake. Nature 2010;464:917-21.

28 Ersoy C, Kiyici S, Budak F, Oral B, Guclu M, Duran C et al. The effect of metformin treatment on VEGF and PAI-1 levels in obese type 2 diabetic patients. Diabetes Res Clin Pract 2008;81:56-60.

29 Dullaart RP, Oomen PH, Sluiter WJ. Circulating vascular endothelial growth factor is unaffected by acute hyperglycemia and hyperinsulinemia in type 1 diabetes mellitus. Eur J Intern Med 2007;18:193-5.

\section{Supporting Information}

Additional Supporting Information may be found in the online version of this article:

Data S1. Materials.

Please note: Wiley-Blackwell are not responsible for the content or functionality of any supporting materials supplied by the authors. Any queries (other than missing material) should be directed to the corresponding author for the article. 


\section{ONLINE SUPPLEMENTARY MATERIAL}

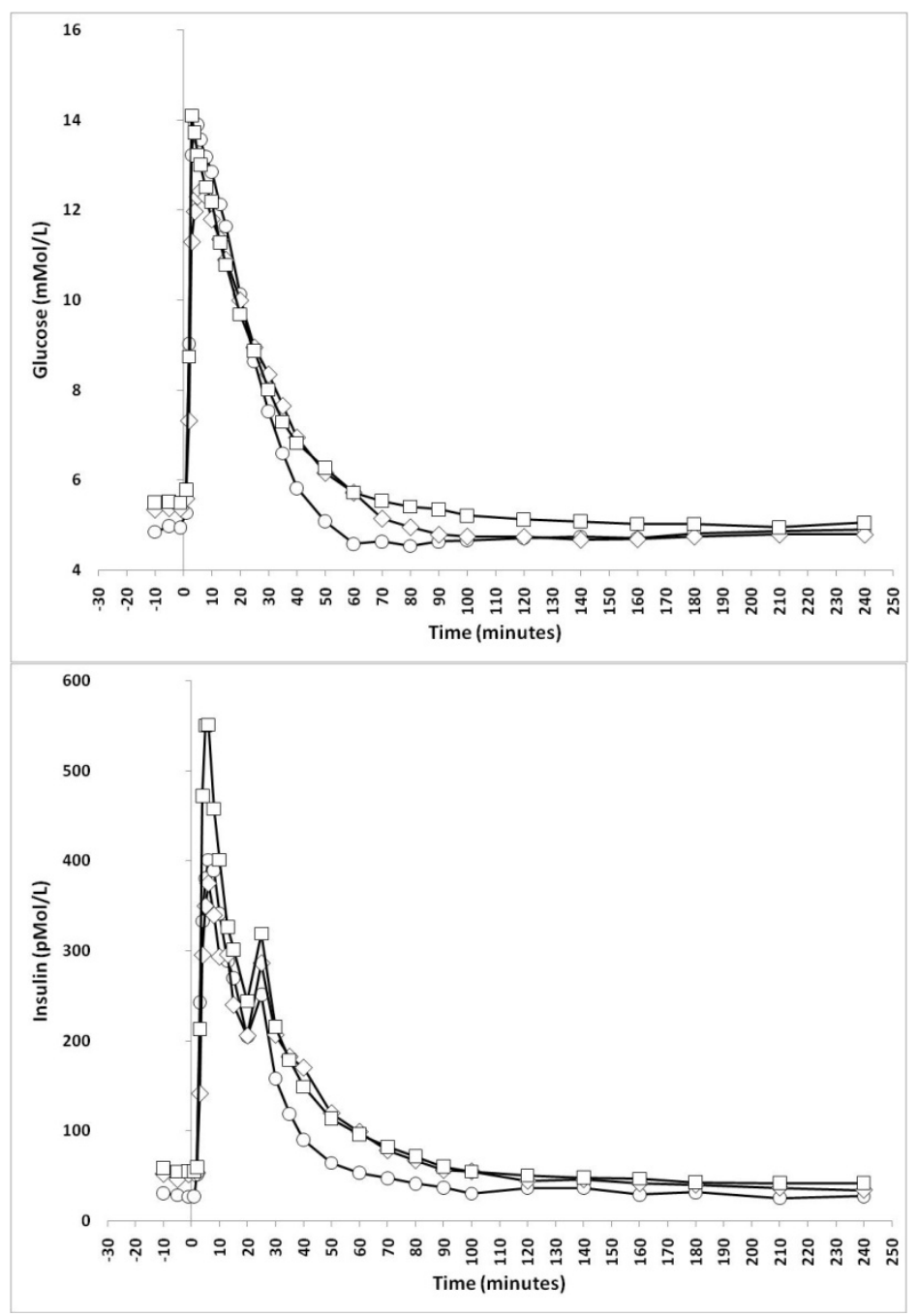

Figure S1: Plasma glucose and insulin concentrations during a FSIVGTT in normal weight and obese subjects divided according to the diagnosis of MetSynSymbols are: control group (0); obese NO-MetSyn $(\diamond)$; obeseMetSyn ( $\square$ ). 


\section{Exclusion Criteria}

Participants were excluded if they had any of the following: history of substance abuse or alcoholism; allergy or intolerance to intervention foods; pregnancy or breastfeeding; professional athletes; weight change more than $3 \mathrm{~kg}$ in the last month; diagnosis of malignancy; chronic and acute metabolic and inflammatory conditions likely to interfere with the study outcome; severe anemia $(\mathrm{Hb}<10 \mathrm{mg} / \mathrm{dL})$; type 1 diabetes and subjects with T2D if treated with insulin and/or oral hypoglycaemic agents; weight loss medications; drugs having an effect on either NO production or on insulin sensitivity (corticosteroids, phenytoin, erythromycin, sildenafil, diuretics, laxatives, anticoagulants, antacids, nitrate derived agents, steroids, statins and any other anti-dyslipidaemic agent, including fibrates and nicotinic acid); Subjects taking antihypertensive agents, hormonal replacement therapies (estrogens, thyroxine, progesteron) and psychiatric drugs (antidepressants, sedatives, antipsychotics) were excluded if the dose had been started/changed in the previous six months. 


\section{Dietary Plan}

\section{Low Nitrate Diet (ONT method)}

It was essential to modify the diet to restrict $\mathrm{NO}_{3}{ }^{-}$intake for of both methods. Since $\mathrm{NO}_{3}{ }^{-}$ occurs in both plants and water it is a natural component of human diet. Nitrate is used as a preservative in cured meat but fresh meat contains little $\mathrm{NO}_{3}{ }^{-}$. Fruit and grains have a small impact on dietary intake of $\mathrm{NO}_{3}{ }^{-}$. The diet and the meals used in this study were prepared on the basis of a recent report on $\mathrm{NO}_{3}{ }^{-}$content in food products ${ }^{1}$. Subjects were advised to avoid all vegetables, cooked and raw, and all tomato based products (spaghetti, pizza sauces, ketchup, tomato juice), potatoes, cured meat, seafood and fish, cheese, wines, beer, alcohol and tap water. Nitrate free water was provided during the study. On this protocol $\mathrm{NO}_{3}{ }^{-}$intake was expected to be $<30 \mathrm{mg} / \mathrm{day}^{2}$.

\section{Very low nitrate meals (ONT method)}

The meals were served to each participant before the start of their 18-hr fasting period. The meals were formulated to have an energy content of $\sim 1400 \mathrm{kcal}$ and provide a $\mathrm{NO}_{3}{ }^{-}$intake $<5$ $\mathrm{mg} /$ meal. The $\mathrm{NO}_{3}{ }^{-}$content of food was derived from a published report on $\mathrm{NO}_{3}{ }^{-}$content in food products ${ }^{1}$.Ingredients with very low $\mathrm{NO}_{3}{ }^{-}$content $(<5 \mathrm{mg} / 100 \mathrm{~g})$ were chosen (pasta, eggs, rice, cereals, oil and spreads, fruit, milk). Low $\mathrm{NO}_{3}{ }^{-}$bottled mineral water $(0.1 \mathrm{mg} / \mathrm{l})$ was used for cooking the meals. Three meals were developed, and the subjects were allowed to choose the one the preferred as this was felt would improve compliance. The McCanceWiddowson food composition tables were used to calculate the energy content of the meals ${ }^{3}$. The nutritional composition of the three meals is described in Table S1a, S1b and S1c. 


\begin{tabular}{|c|c|c|c|c|c|}
\hline $\begin{array}{l}\text { Table S1a: Meal 1 = } \\
\text { Food }\end{array}$ & $\begin{array}{l}\text { Shicken } \\
\text { Grams }\end{array}$ & $\begin{array}{l}\text { ied Rice } \\
\text { Nitrate (mg) /100g }\end{array}$ & Total Nitrate (mg) & Kcal/100g & Total Kcal \\
\hline Chicken Breasts & 100 & 1 & 1 & 155 & 155 \\
\hline Egg & 80 & 0.49 & 0.392 & 151 & 120.8 \\
\hline Rice & 90 & 2.5 & 2.25 & 383 & 344.7 \\
\hline Vegetable oil & 20 & 0 & 0 & 900 & 180 \\
\hline Parmesan cheese & 10 & 1.61 & 0.161 & 400 & 40 \\
\hline Yoghurt & 150 & 0.05 & 0.075 & 82 & 123 \\
\hline Chocolate Mousse & 120 & 0 & 0 & 300 & 360 \\
\hline Chocolate & 40 & 0 & 0 & 520 & 208 \\
\hline Sliced Brown Bread & 40 & 2.5 & 1 & 227 & 90.8 \\
\hline Cream Cheese & 35 & 0 & 0 & 162 & 56.7 \\
\hline Butter & 10 & 0 & 0 & 737 & 73.7 \\
\hline Water & Ad Lib & 0 & 0 & & \\
\hline Coffee/Tea & Ad Lib & 0 & 0 & & \\
\hline Salt & A bit & 0 & 0 & & \\
\hline Pepper & A bit & 0 & 0 & & \\
\hline Total & 695 & 8.15 & 4.878 & 4017 & 1752 \\
\hline
\end{tabular}

\begin{tabular}{|l|c|c|c|c|c|}
\hline Table S1b: Meal 2 $\mathbf{l}$ \\
\hline Food & Grams & Nitrate (mg) /100g & Total Nitrate (mg) & Kcal/100g & Total Kcal \\
\hline Chicken Breasts & 100 & 1 & 1 & 155 & 155 \\
\hline Sliced Brown Bread & 80 & 2.5 & 2 & 207 & 165.6 \\
\hline Mayo & 15 & 0 & 0 & 700 & 105 \\
\hline Yoghurt & 150 & 0.05 & 0.075 & 82 & 123 \\
\hline Chocolate Mousse & 120 & 0 & 0 & 300 & 360 \\
\hline Chocolate & 40 & 0 & 0 & 520 & 208 \\
\hline Sliced Brown Bread & 80 & 2.5 & 2 & 227 & 181.6 \\
\hline Cream Cheese & 35 & 0 & 0 & 162 & 56.7 \\
\hline Butter & 20 & 0 & 0 & & 737 \\
\hline Water & Ad Lib & 0 & 0 & & 147.4 \\
\hline Coffee/Tea & Ad Lib & 0 & 0 & & \\
\hline Salt & A bit & 0 & 0 & & \\
\hline Pepper & A bit & 0 & $\mathbf{6 . 0 5}$ & $\mathbf{3 0 9 0}$ & $\mathbf{1 5 0 2 . 3}$ \\
\hline Total & $\mathbf{6 4 0}$ & & & & \\
\hline
\end{tabular}

\begin{tabular}{|c|c|c|c|c|c|}
\hline Food & Grams & Nitrate (mg) /100g & Total Nitrate (mg) & Kcal/100g & Total Kcal \\
\hline Egg & 160 & 0.49 & 0.784 & 151 & 241.6 \\
\hline Sliced Brown Bread & 80 & 2.5 & 2 & 207 & 165.6 \\
\hline Mayo & 15 & 0 & 0 & 700 & 105 \\
\hline Yoghurt & 150 & 0.05 & 0.075 & 82 & 123 \\
\hline Chocolate Mousse & 120 & 0 & 0 & 300 & 360 \\
\hline Chocolate & 40 & 0 & 0 & 520 & 208 \\
\hline Sliced Brown Bread & 80 & 2.5 & 2 & 227 & 181.6 \\
\hline Cream Cheese & 35 & 0 & 0 & 162 & 56.7 \\
\hline Butter & 20 & 0 & 0 & 737 & 147.4 \\
\hline Water & Ad Lib & 0 & 0 & & \\
\hline Coffee/Tea & Ad Lib & 0 & 0 & & \\
\hline Salt & A bit & 0 & 0 & & \\
\hline Pepper & A bit & 0 & 0 & & \\
\hline Total & 700 & 5.54 & 4.859 & 3086 & 1589 \\
\hline
\end{tabular}

Participants were invited to eat as much as they could. Food was weighted before and after consumption for the calculation of energy and nitrate intake. On average, volunteers ingested $1406 \pm 322 \mathrm{kcal}$ and $4.39 \pm 0.56 \mathrm{mg}$ of energy and nitrate, respectively. 


\section{References}

1. European Prospective Investigation On Cancer, E. FOOD CONTENT OF POTENTIAL CARCINOGENS Nitrates, nitrites, nitrosamines, heterocyclic amines and polycyclic aromatic hydrocarbons. (EPIC, 2004).

2. L'Hirondel, J. Nitrate and Man: Toxic, Harmless Or Beneficial?, (CABI, Walllingford, UK, 2002).

3. Holland, B., Welch AA, Unwin ID, Buss DH, Paul AA, Southgate D. McCance and Widdowson's the composition of food, (The Royal Society of Chemistry, Cambridge, UK, 1991). 